\title{
Evaluation of computer based clinical decision support system and risk chart for management of hypertension in primary care: randomised controlled trial
}

\author{
Alan A Montgomery, Tom Fahey, Tim J Peters, Christopher MacIntosh, Deborah J Sharp
}

\begin{abstract}
Editorial by Jackson
Division of Primary

Health Care,

University of

Bristol, Bristol

BS8 2PR

Alan A

Montgomery

MRC training fellow

Tom Fahey

senior lecturer

Christopher

MacIntosh

technical assistant

Deborah J Sharp

professor

Department of

Social Medicine,

University of Bristol

Tim J Peters

reader in medical

statistics

Correspondence to:

T Fahey

tom.fahey@bristol.

ac.uk
\end{abstract}

BMJ 2000;320:686-90

\author{
Abstract \\ Objectives To investigate the effect of a computer \\ based clinical decision support system and a risk chart \\ on absolute cardiovascular risk, blood pressure, and \\ prescribing of cardiovascular drugs in hypertensive \\ patients. \\ Design Cluster randomised controlled trial. \\ Setting 27 general practices in Avon. \\ Participants 614 patients aged between 60 and 79 \\ years with high blood pressure.
}

Interventions Patients were randomised to computer based clinical decision support system plus cardiovascular risk chart; cardiovascular risk chart alone; or usual care.

Main outcome measures Percentage of patients in each group with a five year cardiovascular risk $\geqslant 10 \%$, systolic blood pressure, diastolic blood pressure, prescribing of cardiovascular drugs.

Results Patients in the computer based clinical decision support system and chart only groups were no more likely to have cardiovascular risk reduced to below $10 \%$ than patients receiving usual care. Patients in the computer based clinical decision support group were more likely to have a cardiovascular risk $\geqslant 10 \%$ than chart only patients, odds ratio $2.3(95 \%$ confidence interval 1.1 to 4.8 ). The chart only group had significantly lower systolic blood pressure compared with the usual care group (difference in means - $4.6 \mathrm{~mm} \mathrm{Hg}$ (95\% confidence interval - 8.4 to -0.8$)$ ). Reduction of diastolic blood pressure did not differ between the three groups. The chart only group were twice as likely to be prescribed two classes of cardiovascular drugs and over three times as likely to be prescribed three or more classes of drugs compared with the other groups.

Conclusions The computer based clinical decision support system did not confer any benefit in absolute risk reduction or blood pressure control and requires further development and evaluation before use in clinical care can be recommended. Use of chart guidelines are associated with a potentially important reduction in systolic blood pressure.

\section{Introduction}

High blood pressure can no longer be viewed as an isolated risk factor for cardiovascular disease. Guidelines from New Zealand for the management of high blood pressure now reflect this view by explicitly including additional risk factors into an overall estimate of absolute cardiovascular risk. ${ }^{12}$ These risk charts are now available on the internet (http://cebm.jr2.ox.ac.uk/ docs/prognosis.html). However, accurate estimation of cardiovascular risk without the use of explicit risk charts or computer based clinical decision support systems is not easy. Health professionals find it difficult to assimilate multiple risk factors into an accurate assessment of cardiovascular risk. ${ }^{34}$ Computer based clinical decision support systems have the advantage of being able to synthesise patient specific information, perform complex evaluations, and present the results to health professionals quickly. ${ }^{5}$ They have been shown to enhance clinical performance in terms of drug dosing and preventive care. ${ }^{5}$ However, their effect on patient outcomes, in particular control of blood pressure, is unclear. $^{56}$

We evaluated the effect of a computer based clinical decision support system and cardiovascular risk chart on patient centred outcomes of absolute cardiovascular risk and blood pressure.

\section{Participants and methods}

A computer based clinical decision support system was written for the two most commonly used practice computing systems (EMIS and AAH Meditel) so that it could be incorporated into routine clinical care. The system is identical to the New Zealand guidelines for the management of hypertension, ${ }^{2}$ except that absolute risk is presented numerically rather than pictorially. The following patient information is required to ascertain absolute cardiovascular risk: sex, age, diabetes, smoking, blood pressure, cholesterol, body mass index, symptomatic cardiovascular disease, family history of ischaemic heart disease, and familial hypercholesterolaemia. The system then calculates the patient's five year risk of a fatal or non-fatal cardiovascular event (newly diagnosed angina, myocardial infarction, coronary heart disease, stroke, or transient ischaemic attack).

\section{Practice randomisation}

We invited all 96 practices in Avon using the EMIS and AAH Meditel computing systems to participate in the study. Practices agreeing to participate in the study were firstly stratified by computer system and then assigned by simple random allocation to use the computer based clinical decision support system and a cardiovascular risk chart (which gives identical information about risk), the risk chart alone, or usual care (no information given about cardiovascular risk). Randomisation was performed with a table of random numbers by a researcher not involved in the study and who was blind to the identity of the practices.

\section{Patients}

All patients aged 60-80 years with a diagnosis of hypertension and a record of having been prescribed antihypertensive drugs in the previous year were eligible. Thirty eligible patients were randomly sampled from each practice list by using either the computer system's built-in sampling facility (EMIS practices) or a 
random sampling program on a personal computer (AAH Meditel practices).

\section{Protocol}

The main study was performed by general practitioners $(n=74)$ and practice nurses $(n=11)$, depending on procedures for management of hypertensive patients within each practice. The general practitioners and nurses were trained to use the computer based clinical decision support system by one of us (AM). Each patient's blood pressure was measured on the day of attendance. Other risk factors were extracted from the patient's notes and cardiovascular risk recorded either automatically (in computer practices) or manually by the general practitioner or nurse (in chart practices). Data were missing only for total and high density lipoprotein cholesterol concentrations. The computer system assumed missing data to have a value representing the lowest addition to absolute risk, and general practitioners and nurses in the chart only practices were instructed to do likewise. A sensitivity analysis was performed for the outcome of absolute cardiovascular risk in which missing values were assigned the median for each trial group. Follow up was at six and 12 months. Because of the nature of the study, neither the doctors and nurses nor the patients were blind to their study group. The study took place from September 1996 to September 1998.

\section{Outcomes}

The primary outcome was the percentage of patients in each group with five year cardiovascular risk $\geqslant 10 \%$. Secondary outcomes were systolic and diastolic blood pressure and prescribing of cardiovascular drugs. Although follow up data were collected at six and 12 months, the primary follow up was at 12 months, and only these results are presented here.

\section{Data analysis}

We managed and analysed data using Stata Statistical Software. ${ }^{7}$ Baseline comparability of the groups was investigated by descriptive statistics. All analyses comparing the groups at follow up were conducted on an intention to treat basis. We used multivariable regression models and adjusted for the value of the outcome variable at baseline and practice computer system. Since randomisation was by practice, we also corrected for clustering using procedures in Stata to derive robust estimates of standard error. Lastly, we tested for an interaction effect of baseline risk level and trial arm.

To assess intensity of treatment, we collected prescribing data for cardiovascular drugs at baseline and six months (there being insufficient time at the end of the trial to repeat the exercise). It was decided in advance to consider this variable in three categories as follows: $0-1 ; 2$; and 3 or more different classes of cardiovascular drugs. The distribution at six months was compared across the three groups by simple $\chi^{2}$ techniques and multinomial logistic regression models that allowed for the corresponding distributions at baseline.

\section{Sample size}

The trial was designed principally to detect a difference between the two intervention arms of the trial, compu- ter based clinical decision support system plus chart versus chart alone. Based on previous work, ${ }^{8}$ we estimated that $55 \%$ of patients would have an absolute cardiovascular risk over five years of $\geqslant 10 \%$. The sample size was designed to detect a difference between these two groups of 20 percentage points in the proportion of patients with five year risk $\geqslant 10 \%$. To allow for randomisation by practice, the sample size was inflated by a factor of two based on an intrapractice correlation coefficient of $0.0551 .{ }^{9}{ }^{10}$ With this inflation factor, $80 \%$ power and two tailed $5 \% \alpha$, the required sample size was 190 in each of the two intervention groups. Twenty seven practices agreed to participate in the trial: 10 were therefore randomly allocated to each of the two intervention arms and seven to the usual care arm. To ensure sufficient numbers of patients with adequate follow up data, 30 patients were randomly sampled from each practice.

\section{Results}

\section{Baseline comparability}

Of 810 randomly selected patients, 95 were excluded before invitation (non-ambulatory patients and those with life threatening illness or who had recently had major surgery (figure)). A further 101 failed to attend the baseline consultation. Of the remaining 614 patients, $552(90 \%)$ and $531(86 \%)$ attended the six and 12 month follow up respectively. Table 1 shows baseline descriptive statistics for the three groups.

\section{Absolute cardiovascular risk}

Table 2 shows unadjusted absolute cardiovascular risk at baseline and 12 month follow up. Adjustment for the clustering effect of using practices as the unit of randomisation did not materially affect the findings and is not reported here. After practice computer sys-

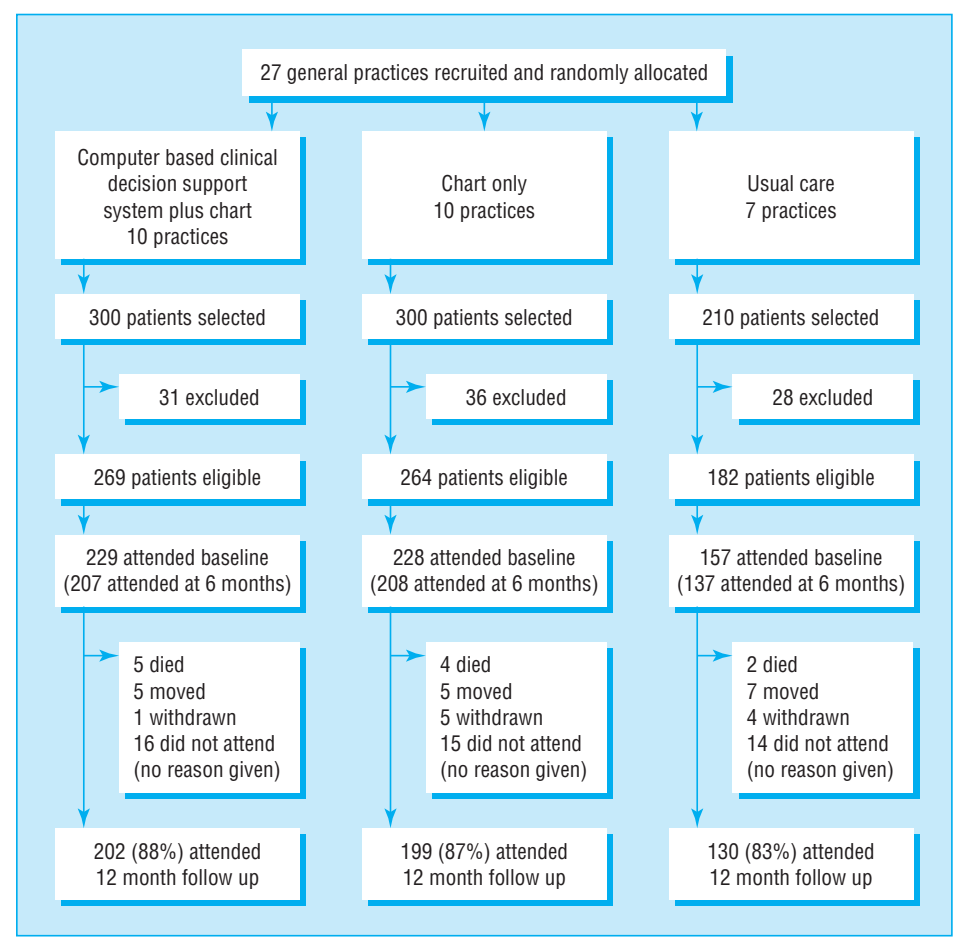

Profile of trial 
Table 1 Baseline characteristics of patients randomised to computer based clinical decision support system plus risk chart, risk chart alone, or usual care. Values are numbers (percentages) of patients unless stated otherwise

\begin{tabular}{lccc} 
& $\begin{array}{c}\text { Computer } \\
\text { support plus } \\
\text { chart }(\mathbf{n = 2 2 9})\end{array}$ & $\begin{array}{c}\text { Chart only } \\
(\mathbf{n = 2 2 8})\end{array}$ & $\begin{array}{c}\text { Usual care } \\
(\mathbf{n}=\mathbf{1 5 7})\end{array}$ \\
\hline Mean (SD) age (years) & $71(6)$ & $70(6)$ & $71(5)$ \\
\hline Women & $123(54)$ & $130(57)$ & $77(49)$ \\
\hline 5 year cardiovascular risk $\geqslant 10 \%$ & $189(83)$ & $198(87)$ & $138(88)$ \\
\hline Mean (SD) absolute 5 year risk (\%) & $18(8)$ & $19(8)$ & $19(9)$ \\
\hline Mean (SD) systolic blood pressure $(\mathrm{mm} \mathrm{Hg})$ & $153(19)$ & $156(19)$ & $158(21)$ \\
\hline Mean (SD) diastolic blood pressure $(\mathrm{mm} \mathrm{Hg})$ & $85(9)$ & $87(9)$ & $86(11)$ \\
\hline Mean (SD) body mass index & $27(4)$ & $28(4)$ & $27(4)$ \\
\hline Mean (SD) total cholesterol $(\mathrm{mmol} / \mathrm{l})$ & $6.0(1.0)(\mathrm{n}=113)$ & $6.1(1.0)(\mathrm{n}=167)$ & $6.0(1.1)(\mathrm{n}=81)$ \\
\hline Mean (SD) high density lipoprotein & $1.3(0.4)(\mathrm{n}=9)$ & $1.3(0.3)(\mathrm{n}=19)$ & $1.2(0.3)(\mathrm{n}=18)$ \\
\hline cholesterol (mmol/) & $32(14)$ & $31(14)$ & $22(14)$ \\
\hline Smoking & $22(10)$ & $27(12)$ & $19(12)$ \\
\hline Diabetes & $8(4)$ & $6(3)$ & $5(3)$ \\
\hline Left ventricular hypertrophy & $17(7)$ & $10(4)$ & $6(4)$ \\
\hline Atrial fibrillation & $24(11)$ & $26(11)$ & $26(17)$ \\
\hline Angina & $8(4)$ & $12(5)$ & $9(6)$ \\
\hline Transient ischaemic attack & $1(1)$ & $4(2)$ & $1(1)$ \\
\hline Angioplasty & $14(6)$ & $8(4)$ & $17(11)$ \\
\hline Peripheral vascular disease & $2(1)$ & $2(1)$ & $4(3)$ \\
\hline Coronary artery bypass graft operation & $15(7)$ & $18(8)$ & $10(6)$ \\
\hline Myocardial infarction & $7(3)$ & $8(4)$ & $11(7)$ \\
\hline Stroke & $52(23)$ & $30(13)$ & $17(11)$ \\
\hline Family history ischaemic heart disease & $27(12)$ & $40(18)$ & $13(8)$ \\
\hline Family history stroke & $4(2)$ & $5(2)$ & $1(1)$ \\
\hline Familial hypercholesterolaemia & &
\end{tabular}

tem and baseline absolute risk were adjusted for by multivariable logistic regression, a significantly greater proportion of patients in the computer based clinical decision support system group were at high risk of a cardiovascular event at follow up compared with those in the chart only group (adjusted odds ratio 2.3, 95\% confidence interval 1.1 to $4.8 ; \mathrm{P}=0.02$ ). There were no significant differences compared with usual care for either the computer based clinical decision support group (1.7, 0.7 to $3.9 ; \mathrm{P}=0.22$ ) or chart only group $(0.7,0.3$ to $1.6 ; \mathrm{P}=0.43)$.

A higher proportion of patients had missing total and high density lipoprotein cholesterol values in the computer support group than the chart group at both baseline (table 1) and follow up. Assigning group medians to missing data did not appreciably change the overall differences between the three groups. Likewise, adjustments for multiple comparisons did not alter the findings between the three groups.

There was evidence of an interaction between the three arms of the trial and level of baseline risk (table 3). Patients at higher baseline risk in both the computer support and chart groups had their absolute cardiovascular risk reduced more effectively than those receiving usual care; the reverse was true in patients at lower baseline risk. When missing cholesterol data were assigned group median values, the interaction disappeared $(\mathrm{F}(2,524)=1.9, \mathrm{P}=0.15)$.

\section{Blood pressure}

Unadjusted means for systolic and diastolic blood pressures at baseline and 12 months are given in table 2. After practice computer system and baseline blood pressure were adjusted for, the only significant difference was that the chart only group had a lower mean systolic blood pressure $(4.6 \mathrm{~mm} \mathrm{Hg}, 95 \%$ confidence interval 0.8 to $8.4 \mathrm{~mm} \mathrm{Hg}, \mathrm{P}=0.02$ ) compared with the usual care group.

\section{Intensity of drug treatment}

Sixteen $(3 \%)$ patients had stopped blood pressure treatment at follow up. Table 4 gives the distributions of the number of cardiovascular drugs being prescribed to the patients at baseline and the six month follow up. When multinomial logistic regression was used to adjust for baseline prescribing, the difference between the trial groups was highly significant $(\mathrm{P}=0.0078)$. Relative to 0-1 classes of cardiovascular drugs, patients in the chart only group were about twice as likely to be prescribed two classes of cardiovascular drugs and over three times as likely to be prescribed three or more classes of drugs (table 5).

Table 3 Change in mean absolute risk at 12 month follow up by baseline risk and trial arm

\begin{tabular}{lccc} 
& \multicolumn{3}{c}{ Mean absolute risk } \\
\cline { 2 - 4 } $\begin{array}{l}\text { Baseline risk } \\
\text { category (\%) }\end{array}$ & $\begin{array}{c}\text { Computer support } \\
\text { plus chart }\end{array}$ & Chart only & Usual care \\
\hline$<10$ & 3.8 & 2.3 & 0.9 \\
\hline $10-19.9$ & 1.5 & 0.7 & 1.8 \\
\hline$\geqslant 20$ & -1.7 & -1.7 & -0.3 \\
\hline All & 0.7 & -0.5 & 0.8
\end{tabular}

Test for interaction between trial arm and baseline risk (using baseline risk as a continuous variable): $F(2,524)=4.88, P<0.01$.

\section{Discussion}

This study shows that in terms of the percentage of patients with a five year cardiovascular risk greater than $10 \%$, neither the computer based clinical decision support system plus chart or chart alone were any better than usual care. The observation that the computer support group had poorer cardiovascular risk reduction than the chart only group is difficult to explain and requires replication. There was some evidence that both the interventions worked more effectively in

Table 2 Unadjusted 5 year risk of cardiovascular event among patients randomised to computer based clinical decision support system plus risk chart, risk chart alone, or usual care and mean systolic and diastolic blood pressure at baseline and 12 month follow up

\begin{tabular}{|c|c|c|c|c|c|c|c|c|c|}
\hline \multirow[b]{2}{*}{ Outcome } & \multicolumn{3}{|c|}{ Computer support plus chart ( $\mathrm{n}=202)$} & \multicolumn{3}{|c|}{ Chart only $(n=199)$} & \multicolumn{3}{|c|}{ Usual care $(n=130)$} \\
\hline & Baseline & 12 months & $\begin{array}{l}\text { Mean (SE) } \\
\text { difference }\end{array}$ & Baseline & 12 months & $\begin{array}{l}\text { Mean (SE) } \\
\text { difference }\end{array}$ & Baseline & 12 months & $\begin{array}{l}\text { Mean (SE) } \\
\text { difference }\end{array}$ \\
\hline \multicolumn{10}{|c|}{5 year cardiovascular risk (No (\%) of patients): } \\
\hline $10-19.9 \%$ & $112(49)$ & $114(56)$ & - & $107(47)$ & $91(46)$ & - & $82(52)$ & $60(46)$ & - \\
\hline$\geqslant 20 \%$ & $77(34)$ & $65(32)$ & - & $91(40)$ & $78(39)$ & - & $56(36)$ & $54(46)$ & - \\
\hline Mean (SD) & $16.0(8.3)$ & $16.7(7.8)$ & $0.65(0.39)$ & $17.9(8.4)$ & $17.5(8.2)$ & $-0.48(0.35)$ & $17.3(8.6)$ & $17.8(9.3)$ & $0.77(0.37)$ \\
\hline Mean (SD) diastolic pressure $(\mathrm{mm} \mathrm{Hg})$ & $85(9)$ & $85(9)$ & $0.36(0.74)$ & $87(9)$ & $86(10)$ & $-1.1(0.78)$ & $86(11)$ & $84(11)$ & $-1.64(1.03)$ \\
\hline
\end{tabular}


higher risk patients compared with usual care (table 3), although this depended on values assigned to missing cholesterol data. Systolic blood pressure was significantly reduced in the chart only group compared with usual care (4.6 mm Hg). This reduction in systolic blood pressure is consistent with significantly increased prescribing of cardiovascular drugs in the chart only group. We found no significant differences in diastolic blood pressure between any of the groups.

\section{Interpretation of findings}

Our results do not support the use of this computer based clinical decision support system in the management of hypertension. The findings are consistent with results of previous studies. ${ }^{56}{ }^{11}$ The benefits of computers are clearer when they are used as administrative aids for detection, registration, and recall. ${ }^{611} 12$

The clinical importance of the reduction in systolic blood pressure in the chart group $(4.6 \mathrm{~mm} \mathrm{Hg})$ should be interpreted in the context of blood pressure reductions achieved in randomised controlled trials of drug treatment. In these studies reductions in systolic blood pressure of $10 \mathrm{~mm} \mathrm{Hg}$ were associated with a reduction of stroke (in relative terms) of $35-40 \%$ and reduction of coronary heart disease of $20-25 \% .{ }^{13}$

One possible reason for the finding that the computer based clinical decision support system does not help manage cardiovascular risk is that the program may have distracted or confused the health professionals in their use of the risk chart. However, all health professionals were specifically trained to use the program. The risk chart strongly depends on visual recognition of risk categories. At the time of the study the computer based clinical decision support system was limited in this respect, since neither computing system was Windows based. More recently developed computer based clinical decision support systems provide visual information about individual cardiovascular risk, comparable information about patients of the same age and sex, and estimates of the likely effect of drug and lifestyle intervention on patients. ${ }^{14}$ Computer systems that are more sophisticated than the one we used may provide better clinical outcomes but will require equally robust evaluation.

Other computer based clinical decision support systems are being developed and are in use in the United Kingdom using the same general practice computer systems as used in this study. ${ }^{15}$ A great deal of investment is taking place in computer based clinical decision support systems on the assumption that patients will benefit. Our results challenge this optimistic view. However, they require replication before final judgment is made.

\section{Study limitations}

Our trial had several limitations. Firstly, the target reduction of 20 percentage points in the proportion of patients at high absolute risk was large, particularly as the only modifiable risk factors are blood pressure, cholesterol, body mass index, and smoking. For this reason, control of blood pressure may be a more suitable and realistic outcome measure. Secondly, the outcome measures were patient based but both interventions were aimed at health professionals. Observational research has shown that health professionals are reluctant to modify cardiovascular prescribing despite
Table 4 Number (percentage) of patients prescribed different numbers of cardiovascular drugs at baseline and six month follow up

\begin{tabular}{|c|c|c|c|c|c|c|}
\hline \multirow{2}{*}{$\begin{array}{l}\text { No of classes } \\
\text { of drugs } \\
\text { prescribed }\end{array}$} & \multicolumn{2}{|c|}{$\begin{array}{l}\text { Computer support plus } \\
\text { chart }(\mathrm{n}=207)\end{array}$} & \multicolumn{2}{|c|}{ Chart only ( $n=208$ ) } & \multicolumn{2}{|c|}{ Usual care $(n=137)$} \\
\hline & Baseline & 6 months & Baseline & 6 months & Baseline & 6 months \\
\hline $0-1$ & $88(43)$ & $81(39)$ & $98(47)$ & $68(33)$ & $58(42)$ & $50(37)$ \\
\hline 2 & $75(36)$ & $74(36)$ & $58(28)$ & $67(32)$ & $45(33)$ & $47(34)$ \\
\hline$\geqslant 3$ & $44(21)$ & $52(25)$ & $52(25)$ & $73(35)$ & $34(25)$ & $40(29)$ \\
\hline
\end{tabular}

Table 5 Multinomial logistic regression analysis of number of types of cardiovascular drugs at six month follow up adjusted for number at baseline

\begin{tabular}{lcc} 
& \multicolumn{2}{c}{ 0dds ratio $(\mathbf{9 5} \% \mathbf{C I})$ compared with $\mathbf{0 - 1}$ classes of drug } \\
\cline { 2 - 3 } & $\mathbf{2}$ drugs & $\geqslant \mathbf{3}$ drugs \\
\hline Chart only & 1 & 1 \\
\hline Computer support plus chart & $0.5(0.2$ to 0.9$)$ & $0.3(0.1$ to 0.6$)$ \\
\hline Usual care & $0.5(0.2$ to 1.0$)$ & $0.3(0.1$ to 0.7$)$ \\
\hline
\end{tabular}

$\chi^{2}(4 \mathrm{df})=13.8 ; P=0.0078$.

persistent high blood pressure. ${ }^{16}$ Thirdly, the computer based clinical decision support system that we used only estimated risk; other aspects of hypertension management such as drug dose and treatment recommendations were not included. ${ }^{17}$ Computer based clinical decision support systems which combine risk estimation and management recommendations for hypertension require further development and evaluation.

\section{Conclusions}

This randomised trial has shown that using a computer based clinical decision support system did not confer any additional benefits compared with chart guidelines and may have impaired the translation of evidence to individual patients. Further studies are required on newer computer based clinical decision support

\section{What is already known on this topic}

Cardiovascular risk in hypertensive patients depends on a wide variety of factors

Guidelines are being increasingly used to help assess risk

Computer based clinical decision support systems have been shown to improve preventive care, but it is unclear whether they affect blood pressure and cardiovascular risk

\section{What this study adds}

Neither a risk chart alone nor a computer based clinical decision support system plus risk chart were any better than usual care in reducing absolute risk of cardiovascular disease

Use of the risk chart alone was associated with a significant reduction in systolic blood pressure and increased prescribing of cardiovascular drugs

Computer based clinical decision support systems require further development and evaluation before introduction into routine practice in primary care 
systems, on how the understanding of absolute risk can influence health professionals' decision making, and how interactive computers can assist patients in decision making, treatment preferences, and adherence to treatment schedules. ${ }^{11} 1819$ Developers of computer based clinical decision support systems should remember that as well as technological development, clinical understanding of the recommendations made by such systems is required.

We thank the 27 Avon practices for participating in this study and Shah Ebrahim for helpful comments on earlier drafts of this paper.

Contributors: The study was conceived and designed by TF, TP, and DS, with additional design input by AM. The computer based clinical decision support system was written by CM. Pilot work was done by AM, TF, and CM. Practices and patients were recruited by AM and TF. AM trained the health professionals in the use of the computer system and risk charts and collected the data. TP, AM, and TF performed the statistical analyses. AM, TF, and TP drafted the paper with contributions from DS and CM. $\mathrm{AM}, \mathrm{TF}$, and TP are the guarantors

Funding: NHS Wales Office of Research and Development, grant number RC016. TF is supported by an NHS R\&D primary care career scientist award.

Competing interests: None declared.

1 Jackson RT, Sackett DL. Guidelines for managing raised blood pressure. BMJ 1996;313:64-5.

2 Core Services Committee. Guidelines for the management of mildly raised blood pressure in New Zealand. Wellington: Ministry of Health, 1994.

3 Chatellier G, Blinowska A, Menard J, Degoulet P. Do physicians estimate

reliably the cardiovascular risk of hypertensive patients? Medinfo $1995 ; 8: 876-9$
4 Grover S, Lowensteyn I, Esrey K, Steinert Y, Joseph L, Abrahamowicz M Do doctors accurately assess coronary risk in their patients? Preliminar results of the coronary health assessment study. BMJ 1995;310:975-8.

5 Hunt D, Haynes RB, Hanna S, Smith K. Effects of computer-based clinical decision support systems on physician performance and patient outcomes. JAMA 1998:280:1339-46.

6 Montgomery AA, Fahey T. A systematic review of the use of computers in the management of hypertension. I Epidemiol Community Health 1998;52:520-5.

7 StataCorp. Stata Statistical Software: Release 6.0. College Station, TX: Stata Corporation, 1999.

8 Fahey TP, Peters T]. A general practice based study examining the absolute risk of cardiovascular disease in treated hypertensive patients. $\mathrm{Br} \mathrm{J}$ Gen Pract 1996;46:655-70.

9 Donner A, Birkett N, Buck C. Randomization by cluster. Am J Epidemiol 1981;114:906-14.

10 Fahey T, Peters TJ. What constitutes controlled hypertension? A patientbased comparison of hypertension guidelines. BMJ 1996;313:93-6.

11 Ebrahim S. Detection, adherence and control of hypertension for the prevention of stroke: a systematic review. Health Tech Assess 1998;2:1-78.

12 Grimshaw JM, Russell IT. Effect of clinical guidelines on medical practice: a systematic review of rigorous evaluations. Lancet 1993;342:1317-22.

13 Collins R, Peto R. Antihypertensive drug therapy: effects on stroke and coronary heart disease. In: Swales JD, ed. Textbook of hypertension. Oxford: Blackwell Scientific, 1994:1156-64.

14 Hingorani A, Vallance P. A simple computer program for guiding management of cardiovascular risk factors and prescribing. BMJ 1999;318:101-5.

15 Purves I. Prodigy interim report. Newcastle: University of Newcastle, Sowerby Unit for Primary Care Informatics, 1996.

16 Berlowitz D, Ash A, Hickey E, Friedman R, Glickman M, Kader B, et al. Inadequate management of blood pressure in a hypertensive population. N Engl J Med 1998;339:1957-63.

17 Randolph AG, Haynes RB, Wyatt JC, Cook DJ, Guyatt GH for Evidence Based Medicine Working Group. Users' guide to the medical literature. XVIII. How to use an article evaluating the clinical impact of a computerbased clinical decision support system. JAMA 1999-282·67-74.

18 Menard J, Chatellier G. Mild hypertension: the mysterious viability of a faulty concept. J Hypertens 1995;13:1071-7.

19 Haynes B, Haines A. Barriers and bridges to evidence based clinical practice. BMJ 1998;317:273-6.

(Accepted 19July 1999)

\title{
Risk assessment in primary prevention of coronary heart disease: randomised comparison of three scoring methods
}

\author{
Christopher G Isles, Lewis D Ritchie, Peter Murchie, John Norrie
}

Editorial by Jackson

Medical Unit,

Dumfries and

Galloway Royal

Infirmary, Dumfries

DG1 4AP

Christopher G Isles

consultant physician

Department of

General Practice

and Primary Care,

University of

Aberdeen,

Aberdeen

AB25 2AY

Lewis D Ritchie

Mackenzie professor of

general practice

Peter Murchie

clinical research fellow

continued over

BMJ 2000;320:690-1
That lipid lowering with statins benefits even those at low risk of coronary heart disease is no longer open to question. The challenge now is for clinicians to strike a balance between what is desirable, affordable, and achievable. As serum total cholesterol concentration alone poorly predicts cardiovascular risk, alternative methods of risk assessment have been proposed. We compared the ability of general practitioners and practice nurses to interpret three of these methods. We chose the revised Sheffield table, ${ }^{1}$ the New Zealand guidelines, ${ }^{2}$ and the joint British chart $^{3}$ because all three included age, sex, smoking and diabetes status, blood pressure, and ratio of total cholesterol to high density lipoprotein cholesterol as part of their risk assessment.

\section{Subjects, methods, and results}

All 37 general practices in Dumfries and Galloway, in Scotland, were randomised to receive the three risk scores in different sequences, each with the same set of 12 case histories. A self nominated general practitioner and nurse in each practice were each asked whether coronary risk exceeded 3\% per year (Sheffield table), whether it exceeded 30\% over 10 years (joint British chart), or whether cardiovascular risk exceeded $20 \%$ over five years (New Zealand guidelines) for each case history. These thresholds were chosen to reflect current practice. $^{45}$ Doctors and nurses also rated each guideline for ease of use and preference, using scales from 1 to $5(5=$ easiest or most preferred).

Accuracy, ease of use, and preference were compared for doctors and nurses separately, first with Freidman's test overall and then with Wilcoxon's signed rank tests on the differences for each subject for pairs of guidelines. $\mathrm{P}$ values reported are unadjusted for multiple comparisons, but the results stand after correction with the Bonferroni method.

Two practices did not have a practice nurse. In another practice the same nurse did not score all three guidelines, and so the results were excluded from the analyses of ease of use and preference. In all, 33/37 doctors and 22/35 nurses scored at least 10 of 12 case histories correctly when using the Sheffield table; corresponding numbers for the New Zealand guidelines were 37 and 33 respectively and for the joint British chart 36 and 34 respectively. There were no significant differences between the three scores for doctors, whereas accuracy among nurses was significantly poorer 